Share Your Innovations through JACS Directory

\title{
Comparative Study on the Direct Current Electrical Conduction in Monolayer and Bilayer Plasma Polymerized Thin Films
}

\author{
M.M. Kamal ${ }^{1, *}$, A.H. Bhuiyan² \\ ${ }^{1}$ Department of Physical Sciences, Independent University, Bangladesh (IUB), Bashundhara R/A, Baridhara, Dhaka - 1229, Bangladesh \\ ${ }^{2}$ Department of Physics, Bangladesh University of Engineering and Technology (BUET), Ramna, Dhaka - 1000, Bangladesh.
}

\section{ARTICLE DETAILS}

\section{Article history:}

Received 04 June 2021

Revised 24 December 2021

Accepted 28 December 2021

Available online 01 February 2022

\section{Keywords:}

Plasma Polymerization

PPPy Thin Film

PPTMA Thin Film

Bilayer Thin Film

Electrical Characterization

DC Conductivity

\begin{abstract}
A B S T RAC T
A comparative study on the nature of direct current electrical conduction in the monolayer and bilayer plasma polymerized thin films has been discussed in this article. The plasma polymerized pyrrole (PPPy) monolayer, plasma polymerized N,N,3,5-tetramethylaniline (PPTMA) monolayer and plasma polymerized pyrrole-N,N,3,5-tetramethylaniline (PPPy-PPTMA) bilayer thin films were deposited at room temperature onto glass substrates by using a parallel plate capacitively coupled glow discharge reactor. The current density-voltage $(\mathrm{J}-\mathrm{V})$ characteristics and the conductivity-voltage $(\sigma-\mathrm{V})$ characteristics have been studied to analyze the direct current conduction mechanism in PPPy, PPTMA monolayer and PPPy-PPTMA bilayer thin films. The observed results have been presented in this paper. The J-V and $\sigma-\mathrm{V}$ characteristics of PPPy and PPTMA monolayer thin films of different thicknesses indicated an increase in electrical conduction and also an increase in conductivity in the films of lower thicknesses. It is also observed that PPTMA thin films are more conductive than that of PPPy thin films. As a result the PPPy-PPTMA bilayer thin films of different thicknesses and different deposition time ratios indicated an increase in conductivity as the proportion of PPTMA is increased in the films. In addition to that, from the J-V and $\sigma-\mathrm{V}$ characteristics of PPPy, PPTMA and PPPy-PPTMA thin films of same thickness, it is observed that the current conduction and the conductivity in the bilayer thin films is less compared to the monolayer thin films. This unusual result has been explained by studying ideal and real bilayer thin films. It is also seen that in the low voltage region, the current conduction obeys Ohm's law while the charge transport phenomenon appears to be the space charge limited conduction (SCLC) in the higher voltage regions.
\end{abstract}

\section{Introduction}

Plasma polymerized thin films are kind of amorphous organic insulating materials, also known as dielectrics, are essential for the proper operation and reliability of all electrical and electronic equipment. The electrical properties of these types of materials is a subject which is inherently interdisciplinary in nature, being closely allied with the mechanical properties of polymers on the one hand, and with the semiconductive properties of inorganic substances on the other.

The direct current (dc) field behavior of a material provides information about the nature of charge carriers, their mobility, conduction mechanism etc. In contrast to metals, in which the electrical field response is one of the electronic conductions, polymers may respond in a more varied manner, and a whole set of delicate electrical effects may be observed. In the study of the dc electrical conduction in the polymer thin films, the current conduction is considered through the film, rather than along the plane of the film and the carriers may either be electronic or ionic in nature. The low field properties are usually Ohmic in nature, but the high field electrical properties cannot be described by a single conduction process. A power law in the form $J \infty V^{n}$, can express the variation of current density with voltage in a material, where $n$ is a power factor. When $n$ is unity, the conduction is Ohmic, and if the value of $\mathrm{n}$ is less or more than unity, then the conduction process is other than Ohmic.

Electronic conduction in amorphous organic insulating solids has received considerable attention in recent years because of its importance in electronic devices. Many reports on the investigation of DC electrical properties of plasma polymerized thin films have been appeared in literature. The influence of electrode material and film thickness on charge transport properties of electrodeposited polypyrrole (PPy) thin films were investigated by Valaski et al. $[1,2]$. They reported that the increase in the

charge mobility and the electrical conductivity were observed by the proper selection of metals with high work function as the electrode and by producing the films with low thickness. Akther and Bhuiyan [3,4] studied the current density-voltage $(\mathrm{J}-\mathrm{V})$ characteristics of plasma polymerized $N, N, 3,5$-tetramethyaniline (PPTMA) thin films of different thicknesses at different temperatures with aluminium (Al) electrodes and reported that the films showed Ohmic behaviour at the lower voltage region and SCLC dominated by exponential trap distribution at the higher voltage region. The electronic conductivity of bilayer aniline-pyrrole thin films were studied by Morales et al. [5]. It was reported that, the bilayer anilinepyrrole polymers had greater electrical conductivity at room temperature than those shown by the separate homopolymers. Kamal and Bhuiyan studied on the optical characterization [6] and direct current electrical characterization [7] of the plasma polymerized pyrrole- $\mathrm{N}, \mathrm{N}, 3,5-$ tetramethylaniline (PPPy-PPTMA) bilayer thin films. The optical characterization [8] and thickness dependent direct current electrical characterization [9] of the PPPy monolayer thin films were also reported by Kamal and Bhuiyan. They also investigated the direct current electrical conduction mechanism of the PPPy monolayer thin films [10] and temperature dependence of DC electrical conduction in PPPy thin films [11]. The comparative study on the structural characterization of the monolayer and bilayer plasma polymerized thin films were reported by them [12]. The dielectric properties of PPPy thin films [13] and alternating current electrical characterization [14] were also studied by Kamal and Bhuiyan.

In this paper a comparative study on the DC electrical conduction of PPPy \& PPTMA monolayer and PPPy-PPTMA bilayer thin films have been presented. The preparation of the monolayer and bilayer thin films, their individual DC electrical conduction properties and the comparison amongst the properties have been described in this article. The current density-voltage $(\mathrm{J}-\mathrm{V})$ characteristics and the DC conductivity-voltage $(\sigma-V)$ characteristics of monolayer and bilayer thin films of different thicknesses with aluminium (Al) electrodes were investigated to study the DC conduction properties in the thin films. The current density-thickness (J- 
d) plots have also been shown to confirm the type of electrical conduction mechanism in the high voltage region.

\section{Experimental Methods}

The PPPy monolayer, PPTMA monolayer and PPPy-PPTMA bilayer thin films were deposited on to chemically cleaned glass substrates at room temperature by using a capacitively coupled glow discharge plasma reactor. The glow discharge system consists of two parallel plate electrodes of stainless steel of diameter and thickness 0.09 and $0.001 \mathrm{~m}$ respectively placed $0.035 \mathrm{~m}$ apart. The glow discharge chamber was evacuated by a rotary pump (Vacuubrand GMBH \& Co, 97877 Wertheim, Germany) and plasma was generated around the substrates which were kept on the lower electrode, using a step up transformer connected to the electrodes with a power of about $30 \mathrm{~W}$ at line frequency.

The monomer pyrrole (Py) and the monomer N,N,3,5tetramethylaniline (TMA) were procured from Aldrich-Chemie D-7924, Steinheim, Germany. The vapor of the monomers was introduced in to the glow discharge reactor through a flow-meter (Glass Precision Engineering, Meterate, England, UK) at the flow rate of about $20 \mathrm{~cm}^{3}$ (STP)/min .

To deposit the bilayer films, pyrrole-monomer has been used as the mother-material and TMA monomer has been deposited in different deposition time ratio after the pyrrole films were formed. The total deposition time was kept $60 \mathrm{~min}$ for all the samples, but the deposition time-ratios of PPPy: PPTMA for the bilayer thin films were (35 min : 25 $\mathrm{min})$, (30 $\mathrm{min}: 30 \mathrm{~min}$ ) and (25 $\mathrm{min}: 35 \mathrm{~min})$ which have produced the films of thicknesses $450 \mathrm{~nm}, 400 \mathrm{~nm}$ and $350 \mathrm{~nm}$ respectively. The PPPy and PPTMA thin films of different thicknesses were also deposited by keeping the deposition parameters (flow-rate, power, vacuum order, etc.) almost same for all samples so that the comparison of the results can be made for various plasma polymerized samples.

For electrical measurements, the lower and upper $\mathrm{Al}$ electrodes were deposited onto the glass substrate and sample respectively i.e. after the lower Al electrode deposited on the substrate, the thin films were formed and then the upper Al electrode was deposited on the film in a similar manner by using an Edward vacuum coating unit E-306A (Edward, UK) at a pressure of about $1.33 \times 10^{-3} \mathrm{~Pa}$ with an effective $\mathrm{Al}$ electrode area of about $10^{-4} \mathrm{~m}^{2}$.

The J-V characteristics of thin films of different thicknesses were studied in $\mathrm{Al} / \mathrm{PPPy} / \mathrm{Al}, \mathrm{Al} / \mathrm{PPTMA} / \mathrm{Al}$ and $\mathrm{Al} / \mathrm{PPPy}-\mathrm{PPTMA} / \mathrm{Al}$ sandwich configurations, with an effective electrode area of $10^{-4} \mathrm{~m}^{2}$ in the voltage range $1.0-30.0 \mathrm{~V}$ at room temperature. The current across the thin films were measured by a high impedance Keithley 614 electrometer and the dc voltage was applied by an Agilent 6545A stabilized dc power supply. The measurements were carried out under dynamic vacuum of about $1.33 \mathrm{~Pa}$ and the temperature was measured by a chromel-alumel thermocouple connected to a digital microvoltmeter 197A (Keithley Instruments, Inc., USA). The thickness of the films was measured by Multiple-Beam interferometry method.

\section{Results and Discussion}

Fig. 1 is the representation of the J-V characteristics for PPPy thin films of thicknesses about $350 \mathrm{~nm}, 400 \mathrm{~nm}$ and $450 \mathrm{~nm}$ which were measured at room temperature in the voltage region $1.0 \mathrm{~V}$ to $30 \mathrm{~V}$. From the figure, it is observed that the current densities (i.e., current conduction) are higher in the films of lower thickness at the same voltage. In our previous investigations [9] it is already reported that the conduction in the plasma polymerized PPPy films is thickness dependent and current densities are found to increase for lower thicknesses in the same applied voltage range and our present results show the similar behaviour.

It is seen that, the curves in the Fig. 1 follow a power law of the form J $\infty$ $\mathrm{V}^{\mathrm{n}}$, where $\mathrm{n}$ is a power index, with two different slopes in the lower and higher voltage regions. The value of slopes at the lower voltage region (1 $\sim 6 \mathrm{~V}$ ) is found to be $0.85<\mathrm{n}<1.15$, indicating a probable $\mathrm{Ohmic}$ conduction, while at the higher voltages $(15 \sim 25 \mathrm{~V})$ the slopes $1.46<\mathrm{n}<2.24$ represent the non-Ohmic conduction.

The J-V characteristics for PPTMA thin films of thicknesses about 350 $\mathrm{nm}, 400 \mathrm{~nm}$ and $450 \mathrm{~nm}$ were recorded at room temperature in the voltage region $1.0 \mathrm{~V}$ to $15 \mathrm{~V}$, and are presented in Fig. 2. This figure also shows the thickness dependent electrical conduction in plasma polymerized thin film. It is to be noted from the figure that the PPTMA thin films are more conductive than PPPy thin films for a certain applied voltage region.

In the Fig. 2 , the value of slopes at the lower voltage region $(1 \sim 4 \mathrm{~V})$ is found to be $0.78<\mathrm{n}<1.06$, indicating a probable Ohmic conduction, while at the higher voltages $(9 \sim 12 \mathrm{~V})$ the slopes $1.89<\mathrm{n}<2.62$ represent the non-Ohmic conduction.

https://doi.org/10.30799/jttr.027.22060101

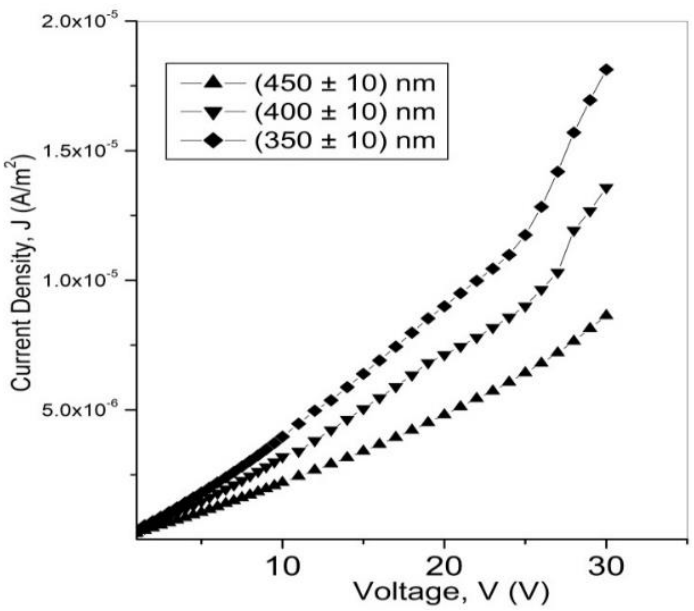

Fig. $1 \mathrm{~J}$-V characteristics for PPPy monolayer thin films of different thicknesses at room temperature

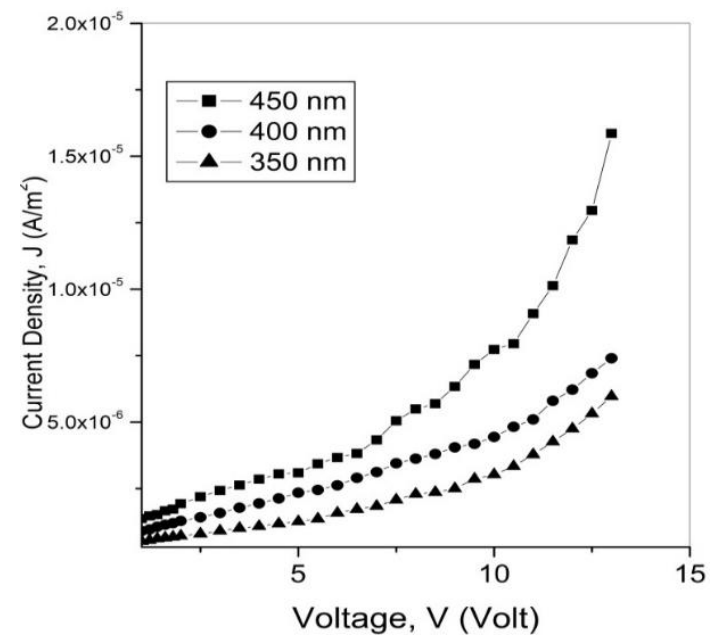

Fig. 2 J-V characteristics for PPTMA monolayer thin films of different thicknesses at room temperature

The J-V characteristics of PPPy-PPTMA bilayer thin films were recorded at room temperature in the voltage region 1.0 to $30 \mathrm{~V}$ and are presented in the Fig. 3. The bilayer thin films were formed in different deposition time-ratios of PPPy and PPTMA as (35 min : $25 \mathrm{~min}$ ), (30 $\mathrm{min}: 30 \mathrm{~min}$ ) and (25 min : $35 \mathrm{~min}$ ) with corresponding thicknesses $450 \mathrm{~nm}, 400 \mathrm{~nm}$ and $350 \mathrm{~nm}$ respectively. It is seen that the thicknesses of the PPPy-PPTMA bilayer thin films were being reduced with the increased proportion of TMA. This is because, the deposition rate of TMA is lower than that of the pyrrole, i.e., if the deposition parameters are remained same then PPTMA thin films are found to be thinner than PPPy thin films. Since the increase in PPTMA in the bilayer thin films causes a decrease in the thickness, therefore increase in the current conduction in bilayer thin films with the increase of PPTMA is not unexpected. From the Fig. 3, as expected, it is observed that the current conduction in the bilayer thin films is increased with the increase of PPTMA in the bilayer structures.

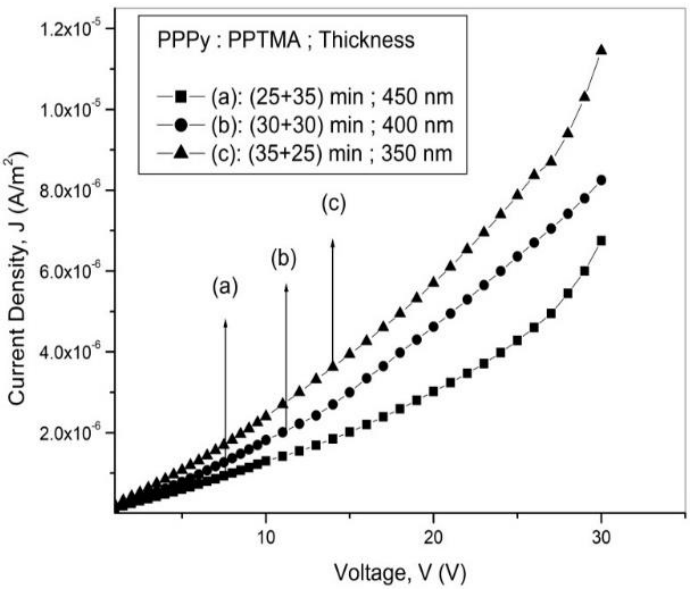

Fig. $3 \mathrm{~J}-\mathrm{V}$ characteristics for PPPy-PPTMA bilayer thin films of different thicknesses at room temperature 
In the Fig. 3, the value of slopes at the lower voltage region $(1 \sim 6 \mathrm{~V})$ is found to be $0.85<\mathrm{n}<1.10$, indicating a probable Ohmic conduction, while at the higher voltages $(15 \sim 25 \mathrm{~V})$ the slopes $1.65<\mathrm{n}<2.45$ represent the non-Ohmic conduction.

It is seen that, the curves in the Figs. 1-3 have two different slopes in the lower and higher voltage regions. At low voltages the J-V characteristics of thin polymer films may follow Ohm's law, provided the transport is not limited by the polymer-electrode interface, and the current density $J$ is the given by the following equation,

$$
J=N q \mu \frac{V}{d}=\sigma \frac{V}{d}
$$

where $q$ is the elementary electric charge, $N$ is the free charge carriers density (in this case, assumed to be positive), $\mu$ is the carrier mobility in the transport band, $d$ is the film thickness, $V$ is the applied voltage, and $\sigma$ is the conductivity [15].

The DC electrical conductivity $\sigma$ of PPPy and PPTMA monolayer thin films, and PPPy-PPTMA bilayer thin films for different thicknesses have been calculated at different voltage from Figs. 1-3 by using Eq.(1). The Fig. 4 shows the plots of $\sigma$ vs. $V$ for PPPy monolayer thin films of different thicknesses.

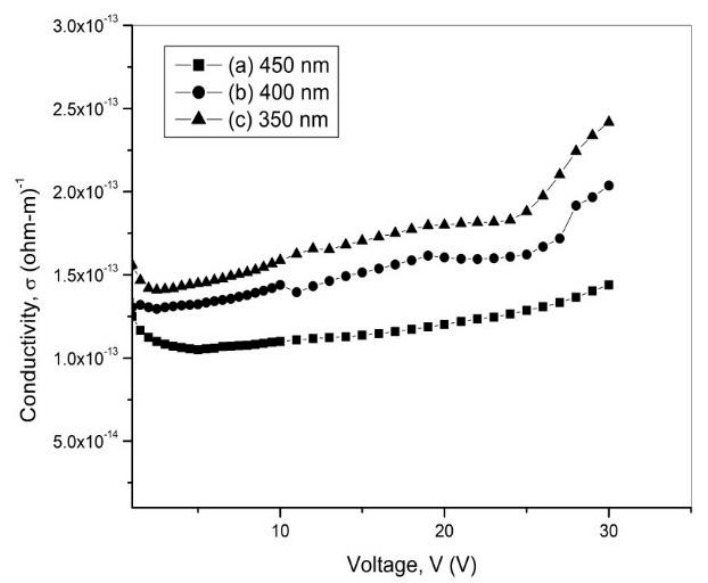

Fig. 4 Plots of DC conductivity $(\sigma)$ vs. voltage $(V)$ for PPPy monolayer thin films of different thicknesses

Though the conductivity $\sigma$ (and consequently the resistivity $\rho$ ) is an inherent physical property of the material, and it is expected that, $\sigma$ or $\rho$ should remain constant in any circumstances, but it is seen from Fig. 4 that, the conductivity is changed with the change in the thickness of the PPPy thin films.

The plots of $\sigma$ vs. $V$ for PPTMA monolayer thin films in Fig. 5 show the similar behaviour. This change in the conductivity for different thicknesses suggests an unexpected change in physical properties during the formation of the plasma polymerized thin films. The most probable reason of this behaviour may be due to better morphological characteristics (lower roughness) and well-ordered neural networks of the films of lower thickness which was reported by Valaski et al. [1, 2]. The thinner films have more structural order due to better homogeneous surfaces, reduced grain size and improve inter chain conduction so that an increased conductivity is usually observed through them.

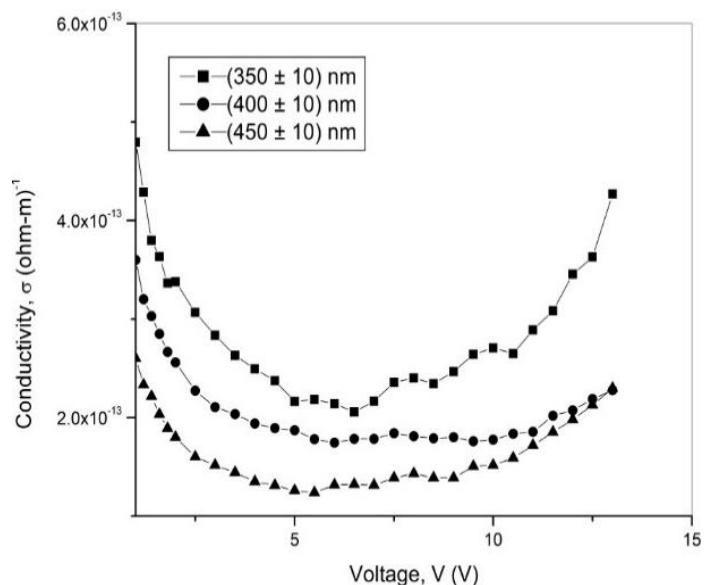

Fig. 5 Plots of DC conductivity $(\sigma)$ vs. voltage (V) for PPTMA monolayer thin films of different thicknesses

https://doi.org/10.30799/jtfr.027.22060101

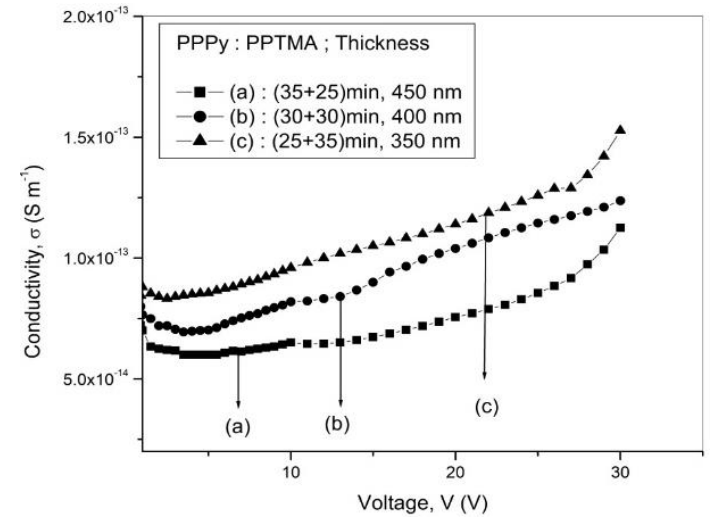

Fig. 6 Plots of DC conductivity ( $\sigma$ ) vs. voltage (V) for PPPy-PPTMA bilayer thin films of different thicknesses

Fig. 6 shows the plots of $\sigma$ vs. $V$ for PPPy-PPTMA bilayer thin films of different thicknesses. It is observed from Fig. 6 that the electrical conductivity of the bilayer thin films is changed as the deposition timeratio is changed, though, it is expected, and for any particular time proportion the physical property like conductivity of the thin film would remain same. The change in the conductivity for different deposition timeratios also suggests a probable change in physical properties during the formation of the bilayer thin films.

The conductivities of PPPy monolayer, PPTMA monolayer and PPPyPPTMA bilayer thin films of thicknesses $450 \mathrm{~nm}, 400 \mathrm{~nm}$ and $350 \mathrm{~nm}$ were calculated at the lower voltage region by using the Eq.(1) and are presented in Table 1.

Table 1 Comparison of DC electrical conductivity $\sigma$ for PPPy, PPTMA and PPPyPPTMA bilayer films of different thicknesses

\begin{tabular}{lll}
\hline Thickness & Sample & Conductivity, $\sigma\left(\mathrm{S} \mathrm{m}^{-1}\right)$ \\
\hline $450 \mathrm{~nm}$ & PPPy monolayer & $(1.1 \pm 0.05) \times 10^{-13}$ \\
& PPTMA monolayer & $(1.3 \pm 0.05) \times 10^{-13}$ \\
& PPPy-PPTMA bilayer & $(6.1 \pm 0.05) \times 10^{-14}$ \\
& (PPPy : PPTMA = $35 \mathrm{~min}: 25 \mathrm{~min})$ & \\
$400 \mathrm{~nm}$ & PPPy monolayer & $(1.3 \pm 0.05) \times 10^{-13}$ \\
& PPTMA monolayer & $(1.8 \pm 0.05) \times 10^{-13}$ \\
& PPPy-PPTMA bilayer & $(7.5 \pm 0.05) \times 10^{-14}$ \\
& (PPPy : PPTMA = 30 min : $30 \mathrm{~min})$ & \\
$350 \mathrm{~nm}$ & PPPy monolayer & $(1.5 \pm 0.05) \times 10^{-13}$ \\
& PPTMA monolayer & $(2.1 \pm 0.05) \times 10^{-13}$ \\
& PPPy-PPTMA bilayer & $(8.7 \pm 0.05) \times 10^{-14}$ \\
& (PPPy : PPTMA = 25 min : $35 \mathrm{~min})$ & \\
\hline
\end{tabular}

These values of $\sigma$ in the Table 1 confirmed our previous discussion that the films of lower thicknesses have higher conductivities. It is also seen from the Table that the conductivity of PPTMA monolayer thin films is greater than that of the PPPy monolayer thin films for any certain thickness. In the case of the PPPy-PPTMA bilayer thin films, the conductivities are found to be smaller than that of its component films. Moreover, it is observed that the conductivity of bilayer films has been increased with the increased proportion of PPTMA. Since the conductivity of PPTMA is higher than that of the PPPy and the presence of greater proportion of PPTMA causes a decrease in the thickness of bilayer films, therefore increase in the current conduction and conductivity in bilayer with the increase of PPTMA is not ambiguous.

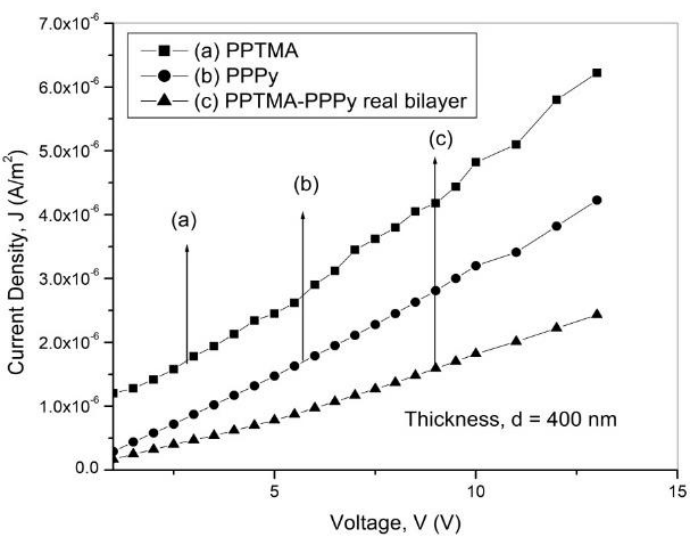

Fig. 7 J-V characteristics for (a) PPTMA monolayer (b) PPPy monolayer and (c) PPPy-PPTMA bilayer thin films at room temperatures $(\mathrm{d}=400 \mathrm{~nm})$ 
For a comparative study of the electrical conductivity of PPPy-PPTMA bilayer thin films with those of the PPPy and PPTMA thin films, the J-V characteristics and $\sigma-\mathrm{V}$ chareacteristics of these three types of films of nearly equal thickness (about $400 \mathrm{~nm}$ ) were recorded at room temperature and are presented in Figs. 7 and 8 respectively.

It is observed from the Fig. 7 that the current conduction in the PPTMA is higher than that in the PPPy thin films and the conduction in the PPPyPPTMA thin films is lower than that of the monolayer thin films. It was also confirmed by estimating the optical band gap of each of the thin films. It is observed that both the direct and indirect energy band gaps are higher for PPPy-PPTMA bilayer thin films than those for PPPy and PPTMA, which was discussed in a separate communication [6].

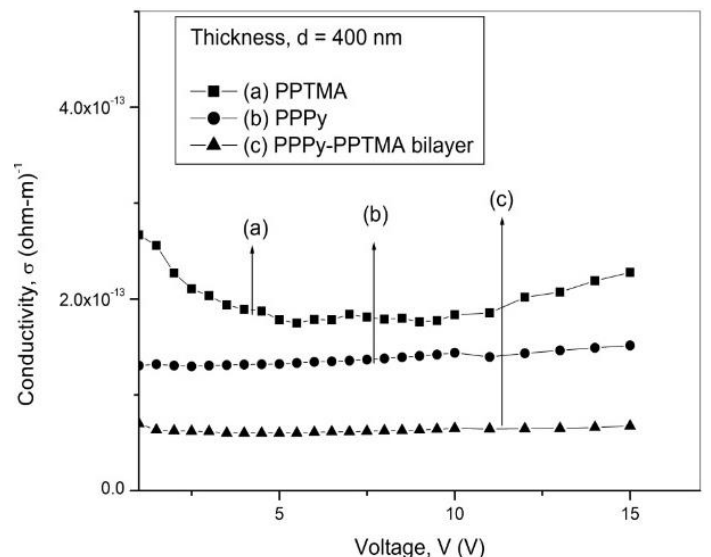

Fig. 8 The $\sigma$-V plots for (a) PPTMA monolayer (b) PPPy monolayer and (c) PPPyPPTMA bilayer thin films at room temperatures $(d=400 \mathrm{~nm})$

From the Fig. 8 and the Table 1, it can be noted that the electrical conductivity of the bilayer thin films is less compared to those of the components which is an unusual behavior for a bilayer or composite thin films. Usually the physical properties of the bilayer without interface effect lie in between those of the constituents, but in the present case, the PPPyPPTMA bilayer thin films show a different nature.

To explain this result Kamal and Bhuiyan proposed [7] that the interface effect between the PPPy and PPTMA thin films in the bilayer structure must be taken in to consideration. For an ideal bilayer thin films the current density, $J^{\prime}$, might be calculated at a fixed applied voltage by the equation [7],

$$
J^{\prime}=\frac{J_{1} J_{2}}{m_{1} J_{2}+m_{2} J_{1}}
$$

where $\mathrm{J}_{\mathrm{i}}$ and $\mathrm{m}_{\mathrm{i}}$ are the current densities and the fraction of the thicknesses of the components respectively. It should be noted that the relationship between the thickness of the component has been evaluated from with an approximation of $m_{1}: m_{2}=$ PPPy : PPTMA $=0.5: 0.5$, with $m=1$ as the bilayer thin film thickness, since the bilayer thin film of Fig. 8 was prepared by depositing the PPPy and PPTMA thin films with a deposition time ratio (30 min : $30 \mathrm{~min}$ ). The theoretically calculated values of $J$ for the ideal bilayer thin film has been plotted in $J-V$ characteristic curves of Fig. 9 to compare with those of the real PPPy-PPTMA bilayer thin films and also with those of the component films.

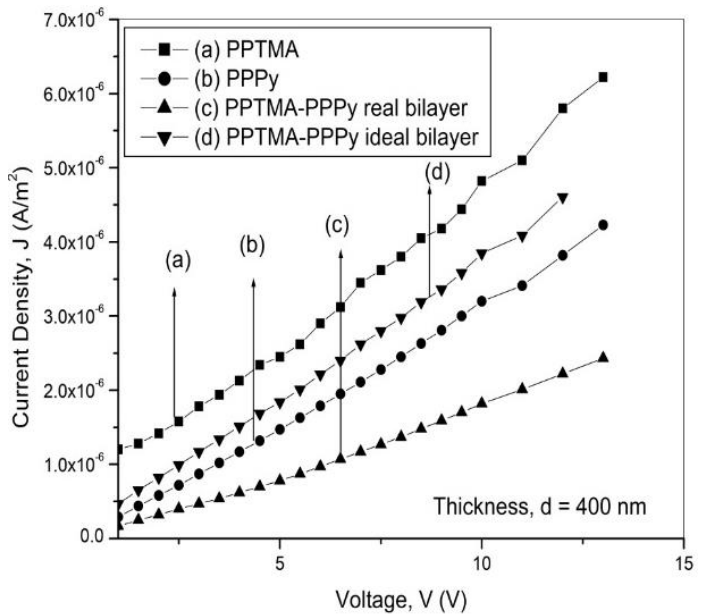

Fig. 9 J-V characteristics for (a) PPTMA (b) PPPy (c) PPPy-PPTMA real bilayer, and (d) PPPy-PPTMA ideal bilayer thin films

https://doi.org/10.30799/jtfr.027.22060101
In Fig. 9, it is observed that the theoretically calculated values of $J$ ' by using Eq.(2) lie in between the values of $J$ of two individual components, but the experimental values of $J$ is less compared to those of its components. This experimental result thus shows a clear violation from the ideal nature of bilayer films. Since both PPPy and PPTMA layers in the bilayer thin film have the same chemical nature as those of the monolayer thin films, therefore the result that the theoretical values of the $J^{\prime}$ of the PPPy-PPTMA ideal bilayer film lie in between those of the components is not unusual. But it is to be noted that when the current density was calculated from the data of the components, the interaction in the interface of the two component thin films was not considered, though this interface might affect the physical properties of the bilayer structure. This type of anomalous effect could give misleading results if they are not recognized or avoided [16]. Therefore, to analyze the electrical behavior of plasma polymerized bilayer thin films, the effect of the interface should be taken into consideration.

The incorporation of oxygen in the polymers is a typical trend of plasma polymer, even though the monomers do not contain oxygen. In our another communication [12] the presence of oxygen by the appearance of the absorption bands at 1850-1603 and $2214 \mathrm{~cm}^{-1}$ in the FTIR spectra of PPPy, PPTMA and PPPy-PPTMA thin films was reported. During the subsequent formation of the thin films by plasma polymerization, there may be adsorbed and/or trapped oxygen in the interface in between the PPPy and PPTMA thin film layers. The oxidation in the interface of the bilayer might affect the physical properties of the thin films. The increase of optical band gap [6] and the decrease of the electrical conductivity of the bilayer thin films may be due to this reason. Furthermore, in the present system, the individual thin films were deposited one over the other to prepare the PPPy-PPTMA bilayer thin films, which may give rise to a system with polymer-polymer complex interface which may not be regular and homogeneous. It is known that in most inhomogeneous polymeric systems, the electronic conductivity is affected by the interface. The electrical conductivity depends upon the movement of adventitious ions generated either by impurities centers or induced impurities [17]. The crosslinking in the interfaces should be also taken into consideration in this system, which may cause less electrical conduction.

At the higher voltage region the complex conduction behavior in thin films could be explained usually in terms of electron emission from cathode, i.e. Schottky-Richardson mechanism; or by electron liberation from the traps in the bulk of the material, i.e. Poole-Frenkel (PF) mechanism; the possibilities of tunneling or Fowler-Nordheim mechanism for very thin films and space charge limited conduction (SCLC) etc., [18]. If the applied voltage is increased over a certain value, the injected charge carrier density largely exceeds the free charge density under thermal equilibrium and the system transits to SCLC conditions. In this case, the $J$ can be described by the Eq.(3) [19],

$$
J=\frac{9}{8} \varepsilon \mu \frac{V^{2}}{d^{3}}
$$

where, $\varepsilon$ is the permittivity of the material.

The thickness dependence of current follows the relation $J \alpha d^{-l}$, where $l$ is a parameter depending upon the trap distribution. A slope $l<3$ suggests the possibility of Schottky or PF conduction mechanism and that $l \geq 3$ reveals the possibility of SCLC.

To study the actual conduction mechanism, $J$ is plotted against $d$ of PPPy and PPTMA monolayer, and PPPy-PPTMA bilayer thin films of different thicknessses at voltage of $27 \mathrm{~V}, 12 \mathrm{~V}$ and $27 \mathrm{~V}$ respectively, which are presented in Fig. 10.

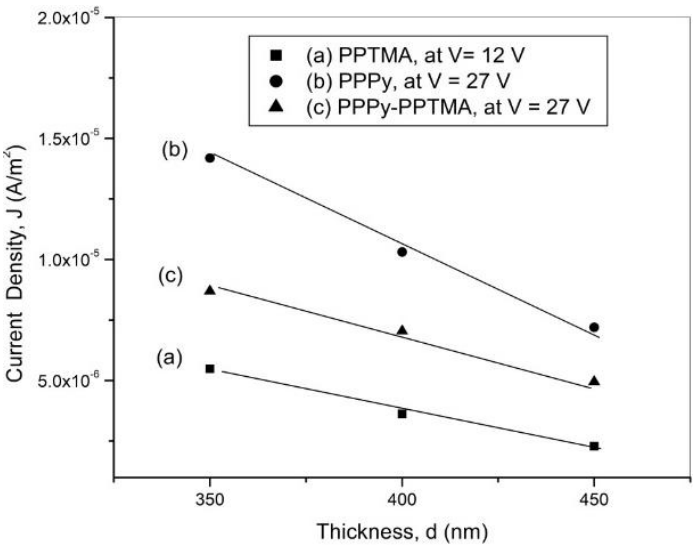

Fig. 10 Plots of J-d for (a) PPTMA monolayer thin films (at V=12 V) (b) PPPy monolayer thin films (at V $=27 \mathrm{~V}$ ) (c) PPPy-PPTMA bilayer thin films (for V $=27 \mathrm{~V}$ ) in the non-Ohmic region 
The linear slope derived from Fig. 10 gives an approximate negative value of 3.4 for all samples, which is much higher than that corresponding to Schottky or PF conduction mechanism. Therefore, the conduction mechanism in PPPy and PPTMA monolayer, and PPPy-PPTMA bilayer thin films is suggested to be SCLC.

\section{Conclusion}

The comparative study of direct current electrical properties of plasma polymerized PPPy and PPTMA monolayer, and PPPy-PPTMA bilayer thin films of different thicknesses in $\mathrm{Al} / \mathrm{PPPy} / \mathrm{Al}, \mathrm{Al} / \mathrm{PPTMA} / \mathrm{Al}$ and $\mathrm{Al} / \mathrm{PPPy}-$ PPTMA / Al sandwich configurations have been presented and the results have been discussed in detail in this article.

From the $J-V$ characteristics of the monolayer and bilayer thin films, it is observed that the films of lower thicknesses (i.e., thinner films) conducts more currents in the same voltage region than that of the films of higher thicknesses (i.e., thicker films). The conductivities $(\sigma)$ of the thinner films were also found to have greater values than that of the thicker films from $\sigma$-V plots. The thinner films have better morphological characteristics (lower roughness) and consequently more structural order due to better homogeneous surfaces, well-ordered neural networks, reduced grain size and improve inter chain conduction etc. and these are the probable reasons of this behaviour of changing physical properties like conductivity (and also the resistivity). It is also observed that the PPTMA monolayer thin films are more conductive than that of the PPPy monolayer films at any certain voltage with same thickness. As a result, in the PPPy-PPTMA bilayer thin films, the current conduction is found to be increased with an increased proportion of PPTMA in the bilayer structure.

The conductivity of PPPy \& PPTMA monolayer and PPPy-PPTMA bilayer thin films of different thickness have also been calculated to compare the conduction nature of these films. The conductivities of PPTMA monolayer thin films are found to be higher than that of the PPPy monolayer films. On the other hand, the conductivities of PPPy-PPTMA bilayer film were found to be lower than both of its component thin films. This results suggest a clear departure of the ideal nature of a bilayer or composite films since in any bilayer/composite material the physical properties lie in between those of the constituents. To explain this unusual result the nature of the ideal (theoretical) and real (experimental) thin films were compared and it is seen that the theoretically calculated values satisfy the ideal condition. The experimental result, on the other hand, suggests that there might be an interface effect which must not be ignored. During the formation bilayer thin films there might grow some inhomogeneous \& irregular complex interfaces between the PPPy and PPTMA thin films in the bilayer structure which affected the electronic conductivity through the bilayer films. To confirm the conduction nature the J-d curves were plotted for all the samples and an Ohmic conduction in the lower voltage and space charge limited conduction in the higher voltages have been observed for PPPy and PPTMA monolayer, and PPPyPPTMA bilayer thin films.

\section{References}

[1] R. Valaski, S. Ayoub, L. Micaroni, I.A. Hümmelgen, Influence of electrode material on charge transport properties of polypyrrole thin films, Thin Solid Films 388 (2001) 171-176.

[2] R. Valaski, S. Ayoub, L. Micaroni, I.A. Hummelgen, Influence of thin thickness on charge transport of electrodeposited polypyrrole thin films, Thin Solid Films 415 (2002) 206-210.

[3] H. Akther, A.H. Bhuiyan, Space charge limited conduction in plasma polymerized N,N,3,5-tetramethylaniline thin films, Thin Solid Films 488 (2005) 93-97.

[4] H. Akther, A.H. Bhuiyan, Electrical and optical properties of plasma polymerized N,N,3,5, -tetramethylaniline thin films, New J. Phys. 7 (2005) 173.

[5] J. Morales, M.G. Olayo, G.J. Cruz, R. Olayo, Synthesis by plasma and characterization of bilayer aniline-pyrrole thin films doped with iodine, J. Polym. Sci. B Polym. Phys. 40 (2002) 1850-1856.

[6] M.M. Kamal, A.H. Bhuiyan, Optical characterization of plasma polymerized pyrrole-N,N,3,5-tetramethylaniline bilayer thin films, J. Appl. Polym. Sci. 121 (2011) 2361-2368.

[7] M.M. Kamal, A.H. Bhuiyan, Direct current electrical characterization of plasma polymerized pyrrole-N,N,3,5-tetramethylaniline bilayer thin films, J. Appl. Polym. Sci. 125 (2012) 1033-1040

[8] M.M. Kamal, A.H. Bhuiyan, Structural and optical characterization of plasma polymerized pyrrole monolayer thin films, Adv. OptoElectron. Mater. 1(2) (2013) 11-17.

[9] M.M. Kamal, A.H. Bhuiyan, Thickness dependent direct current electrical conduction in plasma polymerized pyrrole monolayer thin films, Adv. Mater. Res. 741 (2013) 59-64

[10] M.M. Kamal, A.H. Bhuiyan, Direct current electrical conduction mechanism in plasma polymerized pyrrole thin films, J. Mod. Sci. Tech. 2(2) (2014) 1-9.

[11] M.M. Kamal, A.H. Bhuiyan, Temperature dependence of DC electrical conduction in plasma polymerized pyrrole thin films, J. Mod. Sci. Tech. 4(1) (2016) 36-45.

[12] M.M. Kamal, A.H. Bhuiyan, Comparative study on the structural characterization of monolayer and bilayer plasma polymerized thin films, J. Mod. Tech. Eng. 3(1) (2018) 103-112.

[13] M.M. Kamal, A.H. Bhuiyan, Dielectric properties of plasma polymerized pyrrole monolayer thin films, Global Sci. Tech. J. 6(1) (2018) 27-39.

[14] M.M. Kamal, A.H. Bhuiyan, Alternating current electrical characterization of plasma polymerized pyrrole thin films, Polym. Sci. B 62(3) ( 2020) 264-271.

[15] K.C. Kao, W. Hwang, Electrical transport in solids, Pergamon, Oxford, 1981.

[16] A.R. Blythe, Electrical properties of polymers, Cambridge University Press, Cambridge, London, 1979.

[17] D.A. Seanor, Electrical properties of polymers, Academic Press, Inc, New York, 1982.

[18] H. Yasuda, Plasma polymerization, Academic Press, Inc, New York, 1985

[19] I. Maisel Leon, R. Glang, Hand book of thin film technology, McGraw Hill Book Company, New York, 1970 